\title{
ANR pursues excellence in research, development and delivery
}

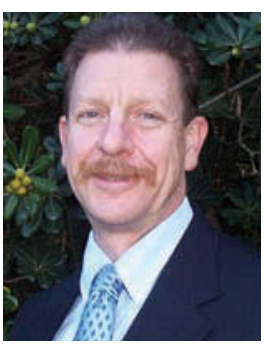

Richard B. Standiford

Associate

Natural Resources
Vice President

Agriculture and

Editor's note: Rick Standiford was appointed associate vice president of ANR in January 2005.

$\mathrm{O}_{\mathrm{t}}$ ver the past 6 months I've been traveling California to meet with UC Agriculture and Natural Resources (ANR) faculty and specialists on the campuses, advisors and staff in county offices, and scientists at research and extension centers. I've also talked with hundreds of people - growers and ranchers, regulators, nutrition professionals, land managers, elected officials, environ-

mental advocates - who rely on the University for research findings, new technologies, and practical know-how to make decisions affecting the economy, health and nutrition, and natural resources.

These visits have been an eye-opener and an enormous source of pride and satisfaction. Even though I had worked with UC for 25 years as a Cooperative Extension forestry specialist, I hadn't fully appreciated the range of agricultural, natural and human-related challenges facing California's growing population. It gives me tremendous satisfaction to know that talented ANR professionals are making a real difference in the lives of millions of Californians.

However, I've also seen the fallout from the state funding cuts we experienced several years ago, resulting in the loss of nearly 350 positions in UC's Agricultural Experiment Station (AES) and Cooperative Extension (UCCE). Our campus- and county-based professionals are working longer hours, taking on greater responsibilities and seeking more effective ways to disseminate research findings to stakeholders.

My travels have confirmed that $\mathrm{UC}$ is the leading public research university in the world, with ANR being a major contributor to this success story. Even with budget cuts, we have nearly 1,000 research and extension academics at UC Riverside, UC Davis, UC Berkeley, county UCCE offices, and research and extension centers statewide. It is unprecedented to have three great campuses in the same state dedicated to the land-grant mission.

The University - through Cooperative Extension — has a world-class system for translating science-based information developed on the campuses and delivering it to end-users across the state. UCCE advisors live and work in local communities and understand the economic, environmental and community development issues facing their neighbors. This focus on local needs is critical in California where demographic diversity, rural-urban conflicts, a broad range of soils and climates, and the production of more than 250 specialty crops and other commodities, create many challenges.

ANR is distinguished by our tradition of interdisciplinary problem-solving and bringing together experts from various fields. At the core of everything we do is a commitment to practice research, development and delivery — or "R, D \& D"
- which means we take basic research from the lab, apply it under strict scientific conditions in the field, then deliver the results to end-users.

But we face a real challenge over the next few years in maintaining these program strengths. The state budget cuts have created gaps in campus- and county-based programs through workforce reductions and the loss of support funds. Not surprisingly, one of the major themes expressed by stakeholders at the listening sessions held across California in early 2004 was for ANR to better focus its mission, direction and resources.

Another common theme was the importance of maintaining viable, locally based UCCE programs. Our stakeholders repeatedly told us that UCCE advisors in the counties are a critical link in translating research and technology developed on our campuses into best management practices adapted for local conditions.

One outcome from the listening sessions was implementation of a broad-based planning process in ANR. A specific goal was to clearly focus our mission and resources in support of high-priority research and extension programs that maximize the public good. The planning process, which will help guide our resource allocation decisions for the next 5 to 10 years, identified 21 core issues (California Agriculture OctDec 2004, p. 178).

As a next step, we funded 28 projects addressing many of the high- and medium-priority core issues identified last fall. The goal was to provide incentives for campus-county collaborative, multidisciplinary, systems-based research and extension efforts. More than 200 ANR academics are now involved in this core-issues grant program.

We also are in the process of rebuilding our county-based UCCE programs, which lost nearly 50 advisor positions. A major step forward was the decision in April to approve 11 new UCCE advisor positions, funded by dollars freed by recent retirements. They address many of our core issues.

For example, we are recruiting an air quality advisor to serve a four-county area in the southern San Joaquin Valley. ANR has strong air quality research programs at Davis, Berkeley and Riverside, and the new CE advisor will help bring science-based solutions and expertise to address agriculture and human health problems in an area that currently fails to meet federal ambient air-quality standards.

The other new positions are a dairy advisor in Tulare County, America's leading dairy county; three vegetable crops advisors; three nutrition, family and consumer sciences advisors; two 4- $\mathrm{H}$ youth development advisors; and a natural resources advisor. We are planning another round of new advisor position approvals later this year, again with funding from retirements.

We in ANR are proud of our partnership with the people of California. We look forward to our continued commitment to quality science, linked directly to solving people's realworld problems. 